Revisão de Literatura - 599 -

\title{
FATORES ASSOCIADOS AO ABANDONO DO TRATAMENTO DA TUBERCULOSE: UMA REVISÃO INTEGRATIVA
}

\author{
Narda Estela Calsin Chirinos ${ }^{1}$, Betina Hörner Schlindwein Meirelles ${ }^{2}$
}

\footnotetext{
${ }^{1}$ Doutoranda do Programa de Pós-Graduação em Enfermagem (PEN) da Universidade Federal de Santa Catarina (UFSC). Professora Principal da Faculdade de Enfermagem da Universidade Nacional do Altiplano. Puno, Perú. E-mail: narda@ccs. ufsc.br

${ }^{2}$ Doutora em Enfermagem. Professora Adjunto do Departamento de Enfermagem PEN/UFSC. Santa Catarina, Brasil. E-mail: betinam@ccs.ufsc.br
}

\begin{abstract}
RESUMO: Revisão integrativa de literatura, em publicações científicas de 2000 a 2009, que objetivou evidenciar conceitos e fatores associados ao abandono do tratamento da tuberculose. Os dados foram coletados do portal da Biblioteca Virtual em Saúde/LILACS e da SciELO, utilizando os descritores: pacientes desistentes do tratamento, recusa do paciente ao tratamento, cooperação do paciente e adesão à medicação, em cruzamento com o descritor tuberculose. A busca originou 219 artigos, que, observados critérios de inclusão e exclusão, resultou em nove artigos completos. A análise dos dados formou as categorias Conceituando abandono de tratamento da tuberculose e Fatores associados ao abandono de tratamento da tuberculose. Considerou-se abandono ao tratamento da tuberculose a interrupção do uso de medicação por 30 dias ou mais. Os fatores associados relevantes foram: sociodemográficos, doenças associadas e assistência em saúde. A equipe de enfermagem/saúde deve focar abordagens de cuidado mais interativas e humanizadas, direcionadas a incrementar a adesão do paciente ao tratamento.
\end{abstract}

DESCRITORES: Tuberculose. Enfermagem. Recusa do pacientes ao tratamento. Adesão à medicação.

\section{FACTORS RELATED TO ABANDONING TUBERCULOSIS TREATMENT: AN INTEGRATIVE REVIEW}

\begin{abstract}
This integrative literature review aimed to simultaneously evidence concepts and to show the factors associated with nonadherence to tuberculosis treatment in scientific publications from 2000 to 2009. Data was collected from the Virtual Health Library/ LILACS and SciELO websites, using the descriptors: Patient giving up treatment, patient treatment refusal, patient cooperation, and adherence to medication, cross-referencing them with the descriptor "tuberculosis". The search resulted in 219 articles. After observing inclusion and exclusion criteria, nine articles were left to compose the sample. Data analysis formed two categories: Conceptualizing abandoning tuberculosis treatment and Factors associated with abandoning tuberculosis treatment. Tuberculosis treatment was considered to be abandoned if the use of such medication was interrupted for thirty days or more. The following associated factors were considered relevant: socio-demographics, related sicknesses/illnesses, and health care. The nursing staff/health care approaches should focus on interactive and more humane care, directed towards increasing adherence to treatment.
\end{abstract}

DESCRIPTORS: Tuberculosis. Nursing. Treatment refusal. Medication adherence.

\section{FACTORES ASOCIADOS CON EL ABANDONO DEL TRATAMIENTO PARA TUBERCULOSIS: UNA REVISIÓN INTEGRADORA}

\begin{abstract}
RESUMEN: Revisión bibliográfica integradora con el fin de demostrar los conceptos y factores asociados con el abandono del tratamiento antituberculoso en publicaciones científicas, de 2000 a 2009. La recolección de los datos se llevo a cabo en el portal de la Biblioteca Virtual en Salud/LILACS y SciELO, según los siguientes descriptores: pacientes que abandonan el tratamiento, rechazo del paciente al tratamiento, cooperación del paciente y adherencia a la medicación, cruzándolos con el descriptor: tuberculosis. La búsqueda condujo a 219 artículos, que al considera criterios de inclusión y exclusión resultó en nueve artículos. Se encontraron las siguientes categorías: conceptualizar el abandono del tratamiento antituberculoso y factores asociados con el abandono del tratamiento antituberculoso. Se consideró como abandono del tratamiento antituberculoso dejar de tomar los medicamentos durante treinta días o más. Los factores relevantes asociados son: sociodemográficos, enfermedades asociadas y atención de la salud. El equipo de enfermería/salud necesita centrarse en enfoques de cuidado más interactivos y humanos para aumentar la adherencia del paciente al tratamiento.
\end{abstract}

DESCRIPTORES: Tuberculosis. Enfermería. Negativa del paciente al tratamiento. Cumplimiento de la medicación. 


\section{INTRODUÇÃO}

Uma das principais preocupações com respeito à tuberculose é a redução das taxas de abandono de tratamento. No Brasil, a taxa de abandono é alta, situa-se em $17 \%$, porém, em muitas regiões, atinge níveis mais elevados: na grande São Paulo a taxa é cerca de $20 \%{ }^{1-2}$ Isso leva ao não rompimento da cadeia de transmissão, pois as pessoas com Tuberculose (TB) que não aderem à terapêutica continuam doentes e permanecem como fonte de contágio. Além disso, o abandono leva à resistência medicamentosa e à recidiva da doença, as quais impõem dificuldades ao processo de cura, aumentando o tempo e o custo do tratamento. ${ }^{3}$

Isso é percebido no percentual insatisfatório de cura da TB, que não ultrapassa $75 \%$ dos casos tratados, embora o Brasil tenha sido o primeiro país a implantar o tratamento de curta duração seis meses - em 1980, com relativo sucesso inicial. ${ }^{4}$

Diagnosticar um paciente com TB pulmonar bacilífera não é o suficiente se não for instituído o tratamento quimioterápico adequado que garanta a sua cura. Para isso, é indispensável o fornecimento ininterrupto e gratuito das drogas e a supervisão das tomadas. $\mathrm{O}$ tratamento dos pacientes bacilíferos é a atividade prioritária de controle da $\mathrm{TB}$, uma vez que permite anular rapidamente as maiores fontes de infecção. ${ }^{4}$

A associação medicamentosa adequada, doses corretas e uso por tempo suficiente, com supervisão da tomada dos medicamentos, são os meios para evitar a persistência bacteriana e o desenvolvimento de resistência às drogas, assegurando, assim, a cura do paciente. ${ }^{4}$

A adesão ao tratamento representa um desafio no controle da TB. Os fatores de proteção interesse em se tratar e nível de informação sobre a doença - e o reconhecimento do uso de droga como fator de risco devem integrar estratégias de cuidado ao doente, buscando reduzir os índices de abandono para recuperação da saúde. ${ }^{2}$

Considera-se caso de abandono de tratamento o doente que, após iniciado o tratamento para $\mathrm{TB}$, deixou de comparecer à unidade de saúde por mais de trinta dias consecutivos, após a data aprazada para o seu retorno. ${ }^{4}$

Vários fatores relacionados ao tratamento, à doença, ao paciente, aos problemas sociais, aos serviços e ao profissional de saúde são apontados como determinantes do problema, e a capacidade dos profissionais de saúde em predizer se um pa- ciente terá ou não uma boa adesão ao tratamento é geralmente baixa. ${ }^{5}$

Os profissionais da saúde tendem a abordar a questão de adesão/não adesão ao tratamento de saúde sob as suas perspectivas, ignorando as do paciente. Eles deixam de considerar a variabilidade e negam a legitimidade dos comportamentos que diferem das suas prescrições. Agindo assim, distanciam-se das ações e razões dos pacientes, julgando-os e rotulando-os, em vez de conhecê-las e entendê-las. ${ }^{5} \mathrm{~A}$ adesão tende a ser menor se a comunicação e a interação entre o paciente e o profissional de saúde é deficiente. ${ }^{6}$

Os fatores de risco para o abandono de tratamento da TB pulmonar são diversos. Tem-se detectado que: baciloscopia negativa no diagnóstico, ausência de trabalho fixo, uso diário de bebida alcoólica, relato de não apresentar melhora clínica durante o tratamento e rejeição ao serviço de saúde, traduzida por sua informação de não voltar ao mesmo serviço e/ou ter procurado outro serviço, ${ }^{3}$ têm certa relevância.

Considerando-se que, para a redução das taxas de abandono de tratamento, é prioritário o conhecimento dos fatores associados a esse abandono, no sentido de reorientar as pessoas em tratamento de TB para práticas e cuidados de saúde, surge a necessidade de conhecer o estado atual do conhecimento sobre o assunto. Assim, tem-se como questão deste estudo: quais os conceitos e os fatores associados ao abandono do tratamento em pessoas com TB, em publicações científicas, na atualidade? Estabelece-se como objetivo deste estudo: evidenciar os conceitos e os fatores associados ao abandono ao tratamento da TB em publicações científicas, no período 2000-2009.

A TB é um problema de saúde prioritário no Brasil, país este que alberga, juntamente com outros 21 países em desenvolvimento, $80 \%$ dos casos mundiais da doença. A reversão desse quadro depende, principalmente, dos profissionais de saúde, que precisam estar atentos e capacitados para informar a população acerca da doença e dos meios de preveni-la, realizar o pronto diagnóstico dos casos suspeitos, iniciar rapidamente o tratamento e acompanhar os pacientes, de modo a garantir-lhes a cura plena. ${ }^{4}$

\section{METODOLOGIA}

Trata-se de uma revisão integrativa da literatura, a fim de deduzir generalizações sobre questões substantivas, a partir de um conjunto 
de estudos diretamente influenciados sobre essas questões. Tais revisões incluem exame das pesquisas para discutir hipóteses, sugestões para novas questões teóricas e identificação de uma pesquisa necessária. ${ }^{7}$

A revisão integrativa é um método de exame específico que resume literaturas empíricas ou teóricas para fornecer uma compreensão mais abrangente de um fenômeno particular. Assim, tem o potencial de apresentar o estado da ciência contribuir para o desenvolvimento da teoria, e tem aplicabilidade direta à prática e à política. ${ }^{8}$

Para ser considerada uma pesquisa, a revisão de literatura deve seguir o mesmo rigor da pesquisa primária. Nesse sentido, para esta revisão, foram consideradas as fases de pesquisa ${ }^{8}$ : identificação do problema e objetivo da pesquisa; pesquisa da literatura com foco sobre o tema a ser estudado; avaliação dos dados aplicando critérios de inclusão e exclusão; análise dos dados extraindo das fontes primárias as características da amostra e método, que, no caso, foi qualquer referência ao conceito de abandono/não adesão e os fatores a que esses estão relacionados. Como última fase, temos a apresentação da síntese, que retrata a construção dos conceitos e seus fatores relacionados.

A busca pela literatura ocorreu na Biblioteca Virtual em Saúde (BVS), nos meses de dezembro 2009 e janeiro 2010. As bases de dados de literatura científica e técnica consultadas foram: Literatura Latino-Americana e de Caribe em Ciências da Saúde (LILACS) e Scientific Electronic Library Online (SciELO). Foram selecionadas essas bases de dados considerando-se o interesse em conhecer a construção desse conhecimento na América Latina, onde a TB tem importante expressão epidemiológica. Os descritores foram selecionados a partir da terminologia em saúde consultada nos Descritores em Ciências da Saúde (DECS-BIREME); são eles: "adesão a medicamento", "recusa do paciente ao tratamento", "pacientes desistentes do tratamento" e "cooperação do paciente"; estes, por sua vez, foram relacionados com o descritor "tuberculose". Inicialmente, a busca considerou as publicações dos últimos cinco anos; porém, optou-se pelo período de 2000 a 2009, que deu maior amplitude ao estudo e resultou em 219 estudos: 32 da base de dados LILACS e 187 das referências na base da SciELO.

Após a leitura dos resumos, segundo pertinência e consistência do conteúdo, foram observados os seguintes critérios de inclusão: estudos disponíveis na íntegra, em open acess, de 2000 a 2009, publicações originais, nas línguas portuguesa, espanhola e inglesa, que adotaram uma abordagem quantitativa, considerando o objetivo do estudo e o protocolo de revisão elaborado previamente. Foram critérios de exclusão: artigos repetidos, artigos não acessíveis em texto completo, resenhas, anais de congresso, artigos de opinião, artigos de reflexão, editoriais, artigos que não abordaram diretamente o tema deste estudo e artigos publicados fora do período de análise. Ao todo, foram excluídos 210 artigos (24 da base de dados LILACS e 186 da base SciELO). Assim, após essa fase, iniciou-se a análise de nove estudos completos.

Foi elaborado um quadro para organização da análise dos artigos contendo os itens: tipo de publicação, ano, fonte, autores, título, tema, método e objetivo. No que se refere aos conceitos e fatores associados ao abandono de tratamento, a análise se fez em termos qualitativos, sumarizando os dados para formar as categorias Conceituando abandono de tratamento da TB e Fatores associados ao abandono de tratamento da TB. Os resultados foram discutidos e sustentados com outras literaturas pertinentes.

\section{RESULTADOS E DISCUSSÃO}

A partir da análise dos textos selecionados, tem-se, quanto ao tipo de publicação, que os nove estudos, apresentados no Quadro 1, são resultados de pesquisas, e todos são nomeados pelos editores como originais.

Com respeito ao ano de publicação, três arti-

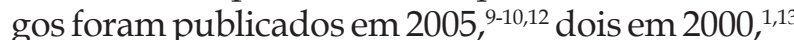
dois em 2007,,15 um em 2001 ${ }^{14}$ e um em 2009, ${ }^{11}$ demonstrando que houve lacunas de publicações na sequência de anos pesquisados.

Quanto aos tipos de estudos, foram assim classificados: estudos descritivos; ${ }^{11,13}$ estudo retrospectivo controlado; ${ }^{1}$ estudo epidemiológico descritivo e sociológico interpretativo; ${ }^{14}$ estudos de caso-controle; ${ }^{2,9}$ coorte histórica; ${ }^{10}$ coorte de $\operatorname{casos}^{15}$ e seccional de base secundária. ${ }^{12}$ Todas as pesquisas adotaram a abordagem quantitativa conforme critério de inclusão, exceto um deles, que teve abordagem quanti-qualitativa, ${ }^{14}$ do qual foram consideradas apenas as análises quantitativas. 
Quadro 1 - Artigos selecionados sobre fatores associados ao abandono de tratamento da tuberculose segundo autores, ano de publicação, título, tipo e objetivo do estudo

\begin{tabular}{|c|c|c|c|c|}
\hline Autores & Ano & Título & $\begin{array}{l}\text { Tipo de } \\
\text { estudo }\end{array}$ & Objetivo \\
\hline $\begin{array}{l}\text { Ribeiro SA, Amado } \\
\text { VM, Camalier AA, } \\
\text { Fernandes MMA, } \\
\text { Schenkman } \mathrm{S}^{1}\end{array}$ & 2000 & $\begin{array}{l}\text { Estudo caso-controle de indica- } \\
\text { dores de abandono em doentes } \\
\text { com tuberculose }\end{array}$ & $\begin{array}{l}\text { Estudo re- } \\
\text { trospectivo } \\
\text { controlado }\end{array}$ & $\begin{array}{l}\text { Comparar características de } \\
\text { pacientes que abandonaram o } \\
\text { tratamento com os que não o aban- } \\
\text { donaram (controle), matriculados } \\
\text { no CS-EPM/Unifesp, no período de } \\
1995 \text { a } 1997 .\end{array}$ \\
\hline $\begin{array}{l}\text { Paixão LMM, Gontijo } \\
\text { ED }^{2}\end{array}$ & 2007 & $\begin{array}{l}\text { Perfil de casos de tuberculose no- } \\
\text { tificados e fatores associados ao } \\
\text { abandono, Belo Horizonte, MG. }\end{array}$ & $\begin{array}{l}\text { Estudo caso } \\
\text { controle }\end{array}$ & $\begin{array}{l}\text { Descrever o perfil dos casos notifi- } \\
\text { cados de tuberculose e analisar os } \\
\text { fatores associados ao abandono do } \\
\text { tratamento. }\end{array}$ \\
\hline $\begin{array}{l}\text { Culqui DR, Grijalva } \\
\text { CG, Reategui SR, } \\
\text { Cajo JM, Suárez LA9 }\end{array}$ & 2005 & $\begin{array}{l}\text { Factores pronósticos de abando- } \\
\text { no del tratamiento antituberculoso } \\
\text { en una región endémica del Perú. }\end{array}$ & $\begin{array}{l}\text { Estudo caso } \\
\text { controle }\end{array}$ & $\begin{array}{l}\text { Identificar fatores preditivos de } \\
\text { abandono do tratamento antituber- } \\
\text { culoso na província de Ica, Perú. }\end{array}$ \\
\hline $\begin{array}{l}\text { Ferreira SMB, Silva } \\
\text { AMC, Botelho } C^{10}\end{array}$ & 2005 & $\begin{array}{l}\text { Abandono do tratamento da } \\
\text { tuberculose pulmonar em Cuiabá. } \\
\text { (MT). Brasil. }\end{array}$ & $\begin{array}{l}\text { Coorte his- } \\
\text { tórica }\end{array}$ & $\begin{array}{l}\text { Identificar os fatores associados } \\
\text { ao abandono do tratamento da } \\
\text { tuberculose pulmonar bacilífera em } \\
\text { Cuiabá, MT. }\end{array}$ \\
\hline $\begin{array}{l}\text { Coelho AGV, Zama- } \\
\text { rioli LA, Perandones } \\
\text { CA, Cuntierre I, } \\
\text { Waldman EA }\end{array}$ & 2009 & $\begin{array}{l}\text { Características da tuberculose } \\
\text { pulmonar em área hiperendêmica } \\
\text { - município de Santos (SP). }\end{array}$ & Descritivo & $\begin{array}{l}\text { Caracterizar o perfil dos pacientes } \\
\text { com tuberculose pulmonar (TBP) no } \\
\text { município de Santos (SP) segundo } \\
\text { fatores biológicos, ambientais e } \\
\text { institucionais. }\end{array}$ \\
\hline $\begin{array}{l}\text { Ferreira AAA, Quei- } \\
\text { roz KCS, Torres KP, } \\
\text { Ferreira MAF, Accioly } \\
\text { H, Alves MSCF }\end{array}$ & 2005 & $\begin{array}{l}\text { Os fatores associados à tubercu- } \\
\text { lose pulmonar e a baciloscopia: } \\
\text { uma contribuição ao diagnóstico } \\
\text { nos serviços de saúde pública. }\end{array}$ & $\begin{array}{l}\text { Seccional } \\
\text { de base } \\
\text { secundária }\end{array}$ & $\begin{array}{l}\text { Verificar os principais fatores asso- } \\
\text { ciados à tuberculose pulmonar e os } \\
\text { resultados da baciloscopia. }\end{array}$ \\
\hline $\begin{array}{l}\text { Oliveira HB, Moreira } \\
\text { Filho } \mathrm{DC}^{13}\end{array}$ & 2000 & $\begin{array}{l}\text { Abandono de tratamento e reci- } \\
\text { diva da tuberculose: aspectos de } \\
\text { episódios prévios, Campinas, SP, } \\
\text { Brasil, 1993-1994. }\end{array}$ & Descritivo & $\begin{array}{l}\text { Identificar as características de } \\
\text { pacientes reingressados no trata- } \\
\text { mento de tuberculose e residentes } \\
\text { na cidade de Campinas, SP, Brasil, } \\
\text { nos anos de } 1993 \text { e } 1994 .\end{array}$ \\
\hline $\begin{array}{l}\text { Lima MB, Mello DA, } \\
\text { Morais APP, Silva } \\
\text { WC }^{14}\end{array}$ & 2001 & $\begin{array}{l}\text { Estudo de casos sobre abandono } \\
\text { do tratamento da tuberculose: } \\
\text { avaliação do atendimento, per- } \\
\text { cepção e conhecimentos sobre a } \\
\text { doença na perspectiva dos clien- } \\
\text { tes (Fortaleza, Ceará, Brasil) }\end{array}$ & $\begin{array}{l}\text { Estudo epi- } \\
\text { demioló-gico } \\
\text { descritivo, } \\
\text { quanti-quali- } \\
\text { tativo }\end{array}$ & $\begin{array}{l}\text { Identificar os fatores que contri- } \\
\text { buem para o abandono do trata- } \\
\text { mento da tuberculose. }\end{array}$ \\
\hline $\begin{array}{l}\text { Albuquerque MFM, } \\
\text { Ximenes RAA, Silva } \\
\text { NL, Sousa WV, Dan- } \\
\text { tas AT, Dantas OMS, } \\
\text { et al. }{ }^{15}\end{array}$ & 2007 & $\begin{array}{l}\text { Fatores associados à falha do } \\
\text { tratamento, abandono e morte } \\
\text { em uma coorte de pacientes de } \\
\text { tuberculose em Recife, Pernam- } \\
\text { buco, Brasil }\end{array}$ & $\begin{array}{l}\text { Coorte de } \\
\text { casos }\end{array}$ & $\begin{array}{l}\text { Investigar a associação de fatores } \\
\text { biológicos, clínicos, sociais, de há- } \\
\text { bitos de vida e acesso aos serviços } \\
\text { de saúde com falência terapêutica, } \\
\text { abandono e óbito, separadamente e } \\
\text { de forma agregada. }\end{array}$ \\
\hline
\end{tabular}

Ao analisar-se a fonte dos artigos, evidenciou-se que a maioria deles ${ }^{1-2,10-15}$ foi publicada em periódicos da área da saúde coletiva e editados no Brasil. Esse resultado mostra que a produção científica sobre o abandono do tratamento da TB na América Latina poderia ser incrementada.

\section{Conceituando abandono de tratamento da TB}

O conceito de abandono de tratamento da $\mathrm{TB}$, presente nas pesquisas, aparece de três formas: 1) Conceito geral, conhecido como o abandono de tratamento não supervisionado: pacientes que 
abandonaram o tratamento são os que ficaram por mais de 30 dias consecutivos sem tomar a medicação contra a TB. $\cdot^{1-2,9-10,15}$ 2) Conceito de abandono de tratamento supervisionado: caracterizado pelo prazo de 30 dias contados a partir da última tomada da droga observada. ${ }^{2}$ 3) Conceito de abandono de tratamento prévio, quando o paciente faltoso por mais de trinta dias, a partir da data agendada, retorna para reiniciar o tratamento ${ }^{13}$ reingressando no sistema de saúde para o tratamento da TB após alta por abandono. ${ }^{10}$

Os estudos determinaram que a característica comum dos conceitos de abandono de tratamento da TB é estar 30 dias ou mais sem uso de medicação. Houve pacientes que abandonaram o tratamento depois de dois a três meses, ${ }^{14}$ outros realizaram o tratamento por tempo inferior a cinco meses. ${ }^{3,10}$

Os abandonos ocorreram predominantemente na primeira metade do tratamento. A melhora clínica do doente, com uma taxa de negatividade de $80 \%$, ocorre ao final do segundo mês. Entretanto, ele continua sendo bacilífero, mas não se sente estimulado para concluir seu tratamento. ${ }^{3}$

O abandono de tratamento supervisionado diferencia-se do não supervisionado, pois exige a supervisão das doses ingeridas dos medicamentos antituberculosos. Assim, identifica-se o abandono no início, permitindo uma ação corretiva imediata. ${ }^{10} \mathrm{~A}$ atual estratégia do tratamento supervisionado (DOTS/TDS) tem como objetivo garantir a adesão ao tratamento, reduzindo o risco de transmissão da doença na comunidade, ${ }^{4}$ e está pautada em cinco pilares, um dos quais é o tratamento diretamente observado. Essa estratégia tem demonstrado aumento significativo na taxa de cura e adesão ao tratamento. ${ }^{16}$ Para favorecer a adesão ao DOT, são oferecidos incentivos aos pacientes, estimulando-os ao cumprimento do tratamento, e a cesta básica foi o item que teve maior impacto para a adesão ao tratamento supervisionado. Em 2003 (ainda sem o incentivo desse item) o percentual de DOT foi $12.6 \%$, e em 2004 esse número chegou a $29,3 \% .{ }^{16} \mathrm{O}$ tratamento supervisionado diminui a taxa de abandono e produz bons resultados quanto à conclusão favorável do tratamento. ${ }^{15}$

No caso do tratamento não supervisionado, o abandono se torna aparente somente quando o paciente falha ao retirar suas medicações, falta à consulta médica ou admite não estar tomando suas medicações prescritas, o que determina demora na intervenção de ação da equipe de saúde. Observa-se que estatisticamente houve 2,41 vezes mais abandono entre os indivíduos que tiveram o tratamento não supervisionado. ${ }^{10} \mathrm{~A}$ ampliação dos atendimentos descentralizados, com implementação do tratamento supervisionado, favorece o controle da $\mathrm{TB}^{2} \mathrm{e}$, com incentivos, garante maior adesão à medicação em oposição ao tratamento não supervisionado.

Cabe salientar que o conceito de abandono/ não adesão ao tratamento da TB mais presente nos artigos é coerente com o conceito preconizado pela Organização Mundial da Saúde e adotado pelo Ministério da Saúde brasileiro: pacientes que ficaram por mais de trinta dias consecutivos sem medicação contra a TB. ${ }^{4}$

\section{Fatores associados ao abandono de tratamento da TB}

Existem vários fatores associados ao abandono do tratamento. A análise dos artigos permitiu a seleção de cinco fatores principais, considerando aspectos sociodemográficos, uso de drogas, aspectos relacionados aos serviços de saúde e ao tratamento da doença, ocorrência de outras doenças, principalmente crônicas, e o cuidado em saúde, os quais serão discutidos a seguir.

Fator 1: os aspectos sociodemográficos interferem na adesão ao tratamento. Os pacientes de sexo masculino abandonam mais o tratamento que os de sexo feminino, com diferenças estatisticamente significativas. ${ }^{1-2,10-12,14}$

Os grupos etários associados ao abandono de tratamento estão na faixa de 30 a 39 anos, ${ }^{13} \mathrm{com}$ idade média de 34,4 e mediana de $32,5,{ }^{2}$ variando entre 20 a 62 anos. ${ }^{11-12,15}$ Cabe salientar que, nos países em desenvolvimento, $80 \%$ dos infectados encontram-se entre 15 e 59 anos; estão, portanto, na faixa de maior produtividade social. A TB afeta principalmente a população economicamente ati$\mathrm{va}^{17-18}$ sobretudo os homens em idade produtiva, acarretando retardo do crescimento econômico, ${ }^{16}$ com prejuízo no desenvolvimento da sociedade, gerando mais pobreza e exclusão social.

A análise da escolaridade do paciente mostra que o analfabetismo e escolaridade inferior ao ensino médio se relacionam com maior probabilidade de abandono de tratamento. ${ }^{9-10,15}$ Interesse em se tratar e informação insuficiente sobre o tratamento proporcionada pelo pessoal de saúde são importantes. ${ }^{2,9}$ Outros fatores que também propiciam o abandono do tratamento são morar longe do centro de saúde, não ter o tempo necessário para buscar o tratamento, associados à relação familiar conflitiva. ${ }^{9}$ 
Semelhantes a este estudo foram os resultados da pesquisa que reafirmam a associação ao abandono de tratamento à baixa escolaridade, à falta de apoio familiar e à falta de orientação no uso dos serviços de saúde. ${ }^{17}$

Fator 2: uso de drogas. Um dos principais motivos de abandono de tratamento da TB está associado ao consumo de drogas,,$^{2,9,12} \mathrm{em}$ especial, ao consumo de álcool ${ }^{1-2,11-12,15}$ e ao tabagismo. ${ }^{1}$ A TB pulmonar está associada ao etilismo e ao tabagismo em $20,6 \%$, ao tabagismo em $19,8 \%$, ao etilismo em $16,6 \% .^{12}$ Os usuários de drogas ilícitas correspondem a $15 \%$ do total dos pacientes com $\mathrm{p}=0,002$, sendo referido o uso de maconha em $33 \%$, cocaína inalatória em $29 \%$, cocaína intravenosa em $17 \%$ e crack em $11 \% .{ }^{1}$ Na maioria desses estudos, pode-se verificar que o consumo de álcool é o fator mais presente nos pacientes com TB, conduzindo-os ao abandono de tratamento. É relevante que o uso diário de bebida alcoólica é fator de risco para o abandono do tratamento e fator relacionado à TB pulmonar respectivamente. ${ }^{3,17-18} \mathrm{O}$ risco de TB ativa é consideravelmente elevado em pessoas que bebem mais de $40 \mathrm{~g}$ de álcool por dia, e/ou tem um transtorno por consumo de álcool. ${ }^{19}$

Fator 3: aspectos relacionados diretamente ao atendimento nos serviços de saúde e ao tratamento da doença. O tratamento prévio de TB está ligado ao abandono. Mesmo em casos de pacientes que participam de grupos educativos de sala de espera, a história de internação prévia está associada em $31,3 \%$, com $p=0,004 .{ }^{1} \mathrm{~A}$ avaliação da sequência do reinício ao tratamento mostrou reiteração, com índice elevado de abandono em $63 \%$. O reingresso ocorreu pela procura espontânea o pelo encaminhamento por outros serviços. Entre os reingressados por abandono prévio, $85,25 \%$ eram pacientes com TB pulmonar, 8,3\% extrapulmonar e $6,5 \%$ apresentaram ambas as formas. ${ }^{13}$ Nos pacientes com história de abandono prévio de TB, a densidade de abandono foi quase três vezes maior do que naqueles considerados como virgens de tratamento. O fato do abandono de tratamentos anteriores traz uma razão de chance de 1,37; num intervalo de confiança de $95 \%(1,06-1,78)$ e $\mathrm{p}=0,017 \cdot{ }^{10} \mathrm{O}$ abandono de tratamento revelou associação ao tratamento prévio, num intervalo de confiança $95 \%(1,51-3,12), p=0,000 .{ }^{11}$

Existem relações entre o insucesso do tratamento para TB e tratamento anterior $(\mathrm{OR}=4,89$; $p<0,001)$ e resistência a duas ou mais drogas antituberculose. O tratamento com drogas adequadas e o acompanhamento dos doentes até a cura são métodos simples e baratos para prevenir o aparecimento de cepas de bacilos resistentes às drogas. ${ }^{18}$

Fator 4: a associação da TB com a ocorrência de outras doenças, principalmente crônicas. A associação da TB pulmonar com a diabetes mellitus foi de $8,7 \%$, com contágio direto ou péssimas condições sanitárias, $10,3 \%$, e com pneumonia não tratada, $6,3 \% .^{12}$ A prevalência de diabetes e coinfecção TB/HIV foi respectivamente de 8,2\% e 16,2\%; esta última vem apresentando um declínio, passou de $20,7 \%$ para $12,9 \%$ entre $2000-2004 .{ }^{11}$ Em $41 \%$ dos pacientes com $\mathrm{TB}$ com registro de sorologia para o HIV, detectou-se $12,95 \%$ de soropositivos sem diferença na forma clínica da TB. ${ }^{2}$ Como patologia associada à TB, merece destaque a aids, com 26,9\% dos pacientes com antecedentes de abandono $(p<$ ou $=0,0013) .{ }^{12}$ A presença de fator de risco para HIV foi relatada por 15\% dos pacientes, desses 73\% abandonaram o tratamento. ${ }^{1}$

A relação retratamento por abandono e coinfecção HIV/TB, história de alcoolismo e internação por complicações foi corroborada em outros estudos. ${ }^{18-19}$ Mesmo com a recomendação da realização de testes de detecção de HIV nos casos de TB, estes foram realizados em menos da metade dos pacientes, com o agravante de que a aids foi a principal causa de óbito entre os pacientes. ${ }^{2}$ Assim, essas pessoas devem ser priorizadas pela assistência nos programas de controle de TB.

Fator 5: os aspectos relacionados ao cuidado em saúde. A humanização da relação dos profissionais de saúde com o usuário pode contribuir como fator de adesão. Com respeito aos profissionais de saúde, os doentes consideram insuficiente a informação sobre o tratamento proporcionada pelo pessoal de saúde $(\mathrm{OR}=4,20) .{ }^{2}$ Horários inadequados, estabelecidos para receber a medicação, relacionam-se com maior probabilidade de abandono (OR=9,95; IC 95\%: 1,77 a 10,02). ${ }^{9}$ As rotinas de atendimento sujeitam os atores a filas, horários inflexíveis e preenchimento de formulários que inviabilizam um atendimento fluente e de qualidade. Ressalta-se que o não cumprimento dos horários de atendimento pelos profissionais reflete na qualidade do exame clínico e na interação com o cliente. ${ }^{14}$ No tratamento prévio, salienta-se: falha na orientação $(24,1 \%)$, falha de agendamento dos retornos $(13,0 \%)$, retirada precoce do medicamento pelo profissional $(12,0 \%)$, dose inferior à indicada $(2,8 \%)$ e internação $(1,9 \%) .^{2}$ Esses resultados mostram o pouco interesse do profissional na adesão do paciente ao tratamento. ${ }^{5} \mathrm{~A}$ ausência de interação e comunicação deficiente entre o paciente e o 
profissional de saúde conduzem à menor adesão ao tratamento, ${ }^{4}$ por exemplo, a falta de repasse de informações de forma abrangente sobre a TB, suas causas e sua forma de transmissão, e a falta de atividades na sala de espera. ${ }^{20} \mathrm{~A}$ adesão ao tratamento relaciona-se com a atuação de equipe multidisciplinar completa no Programa de Controle da Tuberculose. ${ }^{21}$

A perspectiva sobre a questão de adesão do paciente ao tratamento deve mudar. Os profissionais precisam procurar conhecer e entender as perspectivas dos pacientes sobre a adesão e também seus comportamentos. Essa mudança torna-se imprescindível para a adesão da pessoa ao tratamento através da interação. ${ }^{5}$

A interação é parte essencial do cuidado. Este, por sua vez, é objeto da disciplina de enfermagem. ${ }^{22-23} \mathrm{~A}$ apropriação de uma abordagem de cuidado interativo pode permitir maior participação das pessoas com TB e a responsabilidade individual e dos profissionais para o não abandono dos tratamentos.

Ao iniciar o tratamento, a pessoa com TB deveria receber informação detalhada acerca de seu diagnóstico, método usado para chegar a ele, motivos de determinado tratamento, reações adversas potenciais, e consequências da irregularidade do tratamento. Deveria desenvolver-se pelo menos uma sessão educativa sobre seus direitos, ao ingressar e ao sair de alta, que deveria ser vista como uma oportunidade para que cada ex-doente possa potencialmente virar um agente da saúde da comunidade..$^{24}$ Isso corrobora a necessidade de um envolvimento e compromisso dos profissionais de saúde/enfermagem num cuidado mais interativo.

A discussão desses fatores serve de alerta aos profissionais no sentido da necessidade de uma anamnese minuciosa, visando à investigação dos principais fatores associados à $\mathrm{TB}^{12} \mathrm{e}$ à adesão ao tratamento.

\section{CONCLUSÃO}

A literatura pesquisada reforça o conceito de tratamento não supervisionado, no qual a responsabilidade pela adesão ao tratamento é dada à pessoa com TB. Em oposição, no conceito de tratamento supervisionado, uma maior responsabilidade pela adesão à medicação é dada ao profissional de saúde, o que deveria garantir a diminuição da taxa de abandono.

O abandono de tratamento está relacionado a diversos fatores, sobretudo os sociodemográficos, e ocorre principalmente em homens com menor escolaridade; relaciona-se também ao consumo de drogas como o álcool. A associação com outras doenças crônicas, mais especificamente a infecção pelo HIV, está relacionada ao abandono de tratamento da TB.

$\mathrm{O}$ atendimento nos serviços de saúde e experiências anteriores de tratamento da doença estão também relacionados ao abandono do tratamento, demonstrando que a falta de interação e comunicação entre profissionais e pacientes pode levar ao abandono e ao não comparecimento à unidade de saúde.

Assim, entre os fatores relacionados ao abandono do tratamento da TB como contribuintes para a não adesão, salientam-se os relacionados à humanização nos serviços de saúde. Estes nos levam a outras inquietações relacionadas ao tipo de relação que se estabelece entre profissionais e pessoas com TB, muitas vezes marcadas por relações de poder e hegemonia do saber em detrimento das vivências e experiências de quem é sujeito ativo desse processo.

É necessário o desenvolvimento de pesquisas e de novos modelos de cuidado que considere, em seus enfoques, a horizontalidade nas relações entre profissionais e clientes, com diálogo e interação, estabelecendo cuidados que tragam melhores resultados para a compreensão e seguimento do tratamento.

A pesquisa aponta a importância de processos de capacitação da equipe de saúde e enfermagem a fim de rever o paradigma de cuidado e educação adotados, desenvolvendo intervenções diferenciadas, direcionadas a incrementar a adesão do paciente ao tratamento da TB.

\section{REFERÊNCIAS}

1. Ribeiro SA, Amado VM, Camalier AA, Fernandes MAA, Schenkman S. Estudo caso-controle de indicadores de abandono em doentes com tuberculose. J Pneum. 2000 Nov-Dez; 26(6):291-6.

2. Paixão LMM, Gontijo ED. Perfil de casos de tuberculose notificados e fatores associados ao abandono. Belo Horizonte (MG). Rev Saúde Pública. 2007 Abr; 41(2):205-13.

3. Mendes AM, Fensterseifer LM. Tuberculose: porque os pacientes abandonam o tratamento? Bol Pneum Sanitária. 2004 Abr; 12(1):7-38.

4. Ministério da Saúde (BR). Programa Nacional de Controle da tuberculose. manual técnico para controle da tuberculose. $6^{\mathrm{a}}$ ed. Brasília (DF): MS; 2002. 
5. Reiners AAO, Azevedo CR, Vieira MA, Gawlinski AL. Produção bibliográfica sobre adesão/nãoadesão de pessoas ao tratamento de saúde. Ciên Saúde Coletiva. 2006 Jul; 13(2):2294-305.

6. Sousa SS, Silva MGV. Passando pela experiência do tratamento para tuberculose. Texto Contexto Enferm. 2010 Out-Dez; 19(4):636-43.

7. Ganong LH. Integrative review of nursing research. Nurs Health. 1987 Feb; 10: 1-11.

8. Whittemore R, Knafl K. The integrative review: updated metodology. J Adv Nurs. 2005 Dec; 52(5):546-53.

9. Culqui DR, Grijalva CG, Reategui SR, Cajo JM, Suarez LA. Factores pronósticos del abandono del tratamiento antituberculoso em uma región endêmica del Peru. Rev Panam Salud Pública. 2005 Jul; 18(1):14-20.

10. Ferreira SMB, Silva AMC, Botelho C. Abandono de tratamento da tuberculose pulmonar em Cuiabá (MT) Brasil. J Bras Pneum. 2005 Set-Oct; 31(5):427-35.

11. Coelho AGV, Zamarioli L A, Perandones CA, Cuntierre I, Waldman EA. Características da tuberculose pulmonar em área hiperendêmicamunicípio de Santos. J Bras Pneum. 2009 Oct; 35(10):998-1007.

12. Ferreira AAA, Queiroz KCS, Torres KP, Ferreira MAF, Accioly H, Alves MSCF. Os fatores associados à tuberculose pulmonar e a baciloscopia: uma contribuição ao diagnóstico nos serviços de saúde pública. Rev Bras Epidemiol. 2005 Jun; 8(2):142-9.

13. Oliveira HB, Moreira Filho DC. Abandono de tratamento e recidiva da tuberculose: aspectos de episódios prévios, Campinas, SP, Brasil, 1993-1994. Rev Saúde Pública. 2000 Oct; 34(5):437-43

14. Lima MBA, Mello DA, Morais APP, Silva WC. Estudo de casos sobre abandono do tratamento da tuberculose: avaliação do atendimento, percepção e conhecimentos sobre a doença na perspectiva dos clientes. Cad Saúde Pública. 2001 Jul-Ago; 17(4):877-85.

15. Albuquerque MFPM, Ximenes RAA, Silva NL, Souza WV, Dantas AT, Dantas OMS, et al. Fatores associados à falha do tratamento, abandono e morte em uma coorte de pacientes de tuberculose em Recife Brasil. Cad Saúde Pública. 2007 Jul; 23 (7):1573-82
16. Coordenadoria de Vigilância em Saúde da Secretaria Municipal de Saúde da Prefeitura de São Paulo. Informe técnico: avaliação do programa de tuberculose do município de São Paulo [página na Internet]. São Paulo (SP): Prefeitura de São Paulo; 2005 [acesso 2011 Jul 23]. Disponível em: http:// ww2.prefeitura.sp.gov.br//arquivos/secretarias/ saude/vigilancia_saude / publicacao_covisa/0001/ Informe_TB_dez_05.pdf

17. Lozano Salazar JL, Plasencia Asorey C, Ramos Arias D, García Díaz R, Mahíquez Machado LO. Factores de riesgo socioeconómicos de la tuberculosis pulmonar en el municipio de Santiago de Cuba. MEDISAN [online]. 2009 Jan [acesso 2011 Jul 5]; 13(1). Disponível em: http://bvs.sld.cu/revistas/ san/vol13_1_09/san07109.htm

18. Albuquerque MFM, Leitão CCS, Campelo ARL, Souza WV, Salustiano A. Fatores prognósticos para o desfecho do tratamento da tuberculose pulmonar em Recife, Pernambuco, Brasil. Rev Panam Salud Pública. 2001 Jul; 9(6):368-74.

19. Lönnroth K, Williams BG, Stadlin S, Jaramillo E, Dye C. Alcohol use as a risk factor for tuberculosis: systematic review. BMC Public Health. 2008 Aug; 8:289.

20. Santos C. Desigualdades sociais, pobreza e cuidado à saúde: acesso e adesão ao tratamento de tuberculose em uma unidade de saúde do Município do Rio de Janeiro. [dissertação] Rio de Janeiro (RJ): Escola Nacional de Saúde Pública. FIOCRUZ, 2008.

21. Souza MSPL, Pereira SM, Marinho JM, Barreto ML. Características dos serviços de saúde associadas à adesão ao tratamento da tuberculose. Rev Saúde Pública [online]. 2009 [acesso $2011 \mathrm{Jul}$ 24]; 43(6). Disponível em: http://www.scielosp. org / scielo.php? script=sci_arttext\&pid=S0034$89102009000600011 \& \operatorname{lng}=$ en\&nrm=iso.

22. Meleis, A. Theoretical nursing. development and progress. In: Marriner A, Raile M. Modelos y teorías en enfermería. $5^{a}$ ed. Madrid (ES): Ed. Mosby; 2003.

23. Watson J. Enfermagem pós-moderna e futura. Loures (PT): Ed. Técnicas e Científicas; 2002.

24. Castillo OJ. La tuberculosis multirresistente en el Perú. Cuaderno de trabajo 1 [online]. Lima (PE): Foro Salud y Observatorio del derecho a la Salud CIES; 2003 Aug [acesso 2010 Out 28]. Disponível em: http://www.consorcio.org/Observatorio/ publicaciones/jave.pdf 